\title{
Finite element analysis of adhesively bonded composite joints subjected to impact loadings
}

\author{
R. Hazimeh ${ }^{\mathrm{a}, \mathrm{b}}$, G. Challita ${ }^{\mathrm{b}, *}$, K. Khalil $^{\mathrm{b}}$, R. Othman ${ }^{\mathrm{c}}$ \\ a LUNAM Université, Ecole Centrale de Nantes, Institut de Recherche en Génie Civil et Mécanique, UMR CNRS 6183, BP 92101, 44321 Nantes Cedex 3, \\ France ${ }^{\mathrm{b}}$ Equipe MMC, Université Libanaise, Faculté de génie, Campus Hadath, Beyrouth, Lebanon \\ ${ }^{\mathrm{c}}$ Mechanical Engineering Department, Faculty of Engineering, King Abdulaziz University, PO Box 80248, Jeddah 21589, Saudi Arabia
}

\begin{abstract}
The main concern of this paper is to explore the geometrical and material effects on composite double lap joints (DLJ) subjected to dynamic in-plane loadings. Thus, three-dimensional finite element analyses were carried out at quasi-static and impact velocities. The DLJ alone was used for quasi-static case while an output bar was added for impact case. Elastic behavior was assumed for both adhesive and adherends. Average shear stress and stress homogeneity were extracted and compared. It was observed that the adhesive shear stiffness increases the average shear stress. Moreover, it makes the stress heterogeneity more important. On the other hand, higher values of the substrates longitudinal stiffness make the average shear stress higher; whereas, the stress homogeneity in the joint is better achieved for lower substrates' shear stiffness.
\end{abstract}

\section{Introduction}

Adhesive bonding offers many advantages to mechanical joints such as low weight, cost and the ability to join dissimilar materials. It does not cause distortion or weld worms. For this reason, many works have dealt with this subject in the literature. Different aspects were considered such as static, dynamic and impact loadings. Stepped-lap joints under tensile impact were analyzed by Sawa and Ichikawa [1] and showed that peak stress increases with the increase of adherends young's modulus. Mechanism of damage formation in glass-epoxy composite joints under transverse impact was analyzed by Kim et al. [2]. Vaidya et al. [3] found that peak stress for bidirectional composite joint under transverse impact is 10 times higher than under in-plane quasi-static loading. Carlberger and Stigh [4] analyzed impact fracture in aluminum joints under tensile impact and showed that an increase of the strain rate can be achieved in the adhesive layer. Bonded cylinders under shear impact loading were modeled by Sawa et al. [5] to verify experimental split Hokinson pressure bars (SHPB) tests. Silberschmidt et al. [6] studied the effect of impact fatigue on the crack growth of bonded joints. High velocity transverse impact on composite joints was investigated by Park

\footnotetext{
* Corresponding author.

E-mail addresses: Rachad.Hazimeh@ec-nantes.fr (R. Hazimeh) George_Challita@yahoo.com (G. Challita), Khkhalil@ul.edu.lb (K. Khalil), rothman1@kau.edu.sa (R. Othman).
}

and Kim [7], plies delamination were observed at the highest peel and shear stresses. Challita and Othman [8] simulated the SHPB tests on double-lap bonded joints with metal substrates and concluded that the SHPB bar method gives a good estimation of the mean adhesive stress value and not for adhesive average strain and maximum stress and strain; a unified parameter was proposed to correct the SHPB results. Stress wave propagation in epoxy-steel cylinders subjected to impact push-off loads under small strain rates was analyzed by Liao and Sawa [9] and showed that normal stresses increase with the increase of adherend/adhesive stiffness ratio. Liao et al. [10] studied the single-lap joint (SLJ) subjected to impact tensile medium strain rate, as overlap length increases, maximum principal stress decreases while adherends young's modulus and loading rate have the opposite effect.

The aim of this paper is to present a numerical 3D parametric study on the stress distribution inside adhesive layer for composite DLJ under in-plane quasi-static and impact loading cases. Contrarily to Challita and Othman [8], we are dealing here with composite substrates.

\section{Method}

\subsection{Sample geometry}

In this paper, we were interested in the double-lap adhesive joints as depicted in Fig. 1. Since peel stresses are reduced in 
double lap joints comparing to single lap joints, we focused on the shear stress distribution inside the adhesive layer. A compressive rightwards load was applied on the central substrate which yields to a shear stress state in the adhesive layer. First, a reference model was studied. Subsequently, a parametric study considering the different geometrical and material parameters was carried out while maintaining adherends' similarity in the material and orientation.

\subsection{Loading cases}

Following Challita and Othman [8], we are interested in analyzing the accuracy of experimental testing of double-lap joints by using finite-element simulations. Contrarily to Ref. [8], we are dealing with composite substrates and not metal ones.

In the quasi-static range, the double lap joints are commonly tested by servo-hydraulic machines. The loading rate can be as slow as $0.1 \mathrm{~mm} / \mathrm{min}$. At impact loading rates, the split Hokinson pressure bars system is widely used. Strain rate can be of $10^{4} \mathrm{~s}^{-1}$. The specimen is sandwiched between two bars, termed respectively input and output bar; the incident wave splits into two other waves at the specimen"input bar" interface, a reflecting wave through the first input bar and a transmitted wave through the specimen to the output bar. The reader is referred to Ref. [8] for further details on the SHPB method. Yet, Dharan and Hausser [11] introduced the direct-impact technique, whereas, the input bar is removed and the specimen is directly impacted by the incident bar.

Usually, in a servo-hydraulic mechanical test, the specimen (the double lap joint) is sandwiched between two rigid plates. One

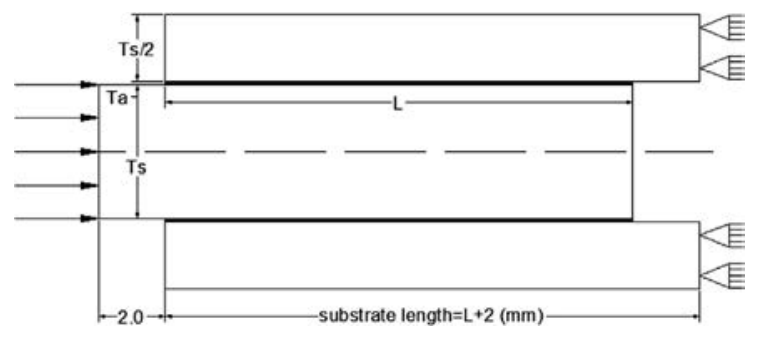

Fig. 1. Sample geometry with width $W$.

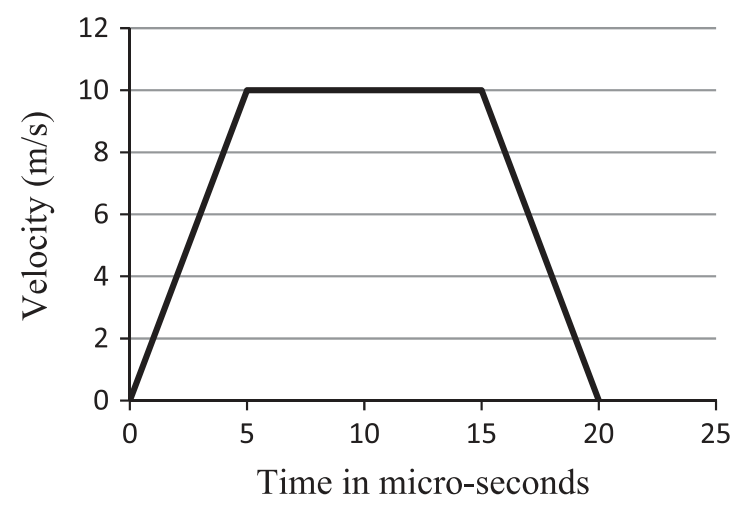

Fig. 2. Impact pulse. plate is moving at almost constant velocity whereas the second one is fixed. In order to simulate such loading case, a $0.1 \mathrm{~mm} / \mathrm{min}$ steady-state velocity was applied on the inner substrate of the joint for a total time of $90 \mathrm{~s}$. Therefore, a total displacement of $0.15 \mathrm{~mm}$ was imposed to the inner substrate by the end of the simulation. This loading case is referred hereafter as the quasistatic case. A second loading case was considered and is referred hereafter as the impact case. It simulates the loading to which a double-lap specimen is submitted during a direct-impact Hopkinson bar test. Therefore, a velocity impact pulse was applied on the inner substrate for a total duration of $20 \mu \mathrm{s}$. The impact pulse is shown in Fig. 2. Similarly to the quasi-static case, an almost total displacement of $0.15 \mathrm{~mm}$ was imposed to the inner substrate at the end of the numerical simulation.

\subsection{Material properties}

Both adhesive and substrates were assumed elastic as suggested by Higuchi et al. [17,18] and Sawa et al. [19] who compared their results to drop weight experiments. Indeed, elastic behavior is valid for elastic-brittle adhesives before failure and for ductile adhesives before yielding. The results of this study should be considered in this framework. Isotropy was assumed for the adhesive. However, as we were interested in composite laminate adherends, orthotropic elasticity was considered for substrates.

In the case of the reference numerical model, the material properties of Polyether ether ketone (PEEK) reinforced with $60 \%$ volume glass fiber were adopted. Moreover, we assumed that fibers are unidirectional and oriented parallel to the load with ply thickness of $0.2 \mathrm{~mm}$. The adhesive is Araldite 2031, black epoxy system suitable for composite bonding. Material properties for reference model are shown in Table 1.

The material properties for the substrates are calculated using the mixing law.

\subsection{Numerical model}

For the quasi-static loading, the sole specimen was modeled with proper boundary conditions. For the impact case, the specimen and the output bar were modeled. Due to symmetry conditions, one-fourth of the system was modeled. The numerical models are shown in Fig. 3.

The commercial software ABAQUS was used with its implicit module for quasi-static case and explicit module for impact case. Tied node-to-surface was used between adhesive and substrates. The C3D8R 8-node solid element was used; each node has 3 degrees of freedom with reduced integration and hourglass control.

Since the thickness of the adhesive is a parameter, and we averaged stresses through the adhesive thickness, an element size of $0.025 \mathrm{~mm}$ through thickness was chosen to build-up any adhesive layer, which corresponds to 4 elements over adhesive thickness for the reference model. A $5 \times 100 \times 25 \mu \mathrm{m}^{3}$ smallest element was used at the joint edges. This mesh size was sufficient to achieve convergence as will be shown in Section 2.6.

Table 1

Material properties [12-16].

\begin{tabular}{|c|c|c|c|c|}
\hline & Young's modulus (GPa) & Poisson's ratio & Rigidity' s modulus (GPa) & Limit stress (MPa) \\
\hline PEEK & $E_{m}=4.1$ & $\nu_{m}=0.4$ & $G_{m}=1.3$ & $\sigma=118$ \\
\hline Glass E & $E_{f}=72$ & $\nu_{f}=0.22$ & $G_{f}=30$ & $\sigma=3300$ \\
\hline Composite ply: $V_{f}=60 \%$ and $V_{m}=40 \%$ & $E_{1}=44.84 E_{2}=E_{3}=9.44$ & $\begin{array}{l}\nu_{12}=\nu_{13}=0.292 \\
\nu_{23}=0.4\end{array}$ & $\begin{array}{l}G_{12}=G_{13}=3.05 \\
G_{23}=3.37\end{array}$ & $\sigma_{R}=2730$ \\
\hline Adhesive (Araldite 2031) & $E=1$ & $\nu=0.4$ & $G=0.35$ & $\sigma=20$ \\
\hline
\end{tabular}


a

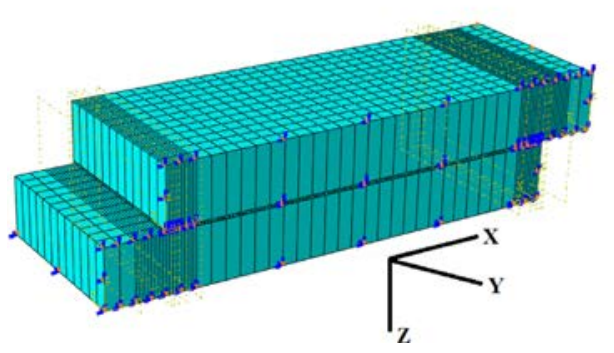

b

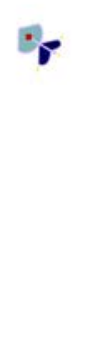

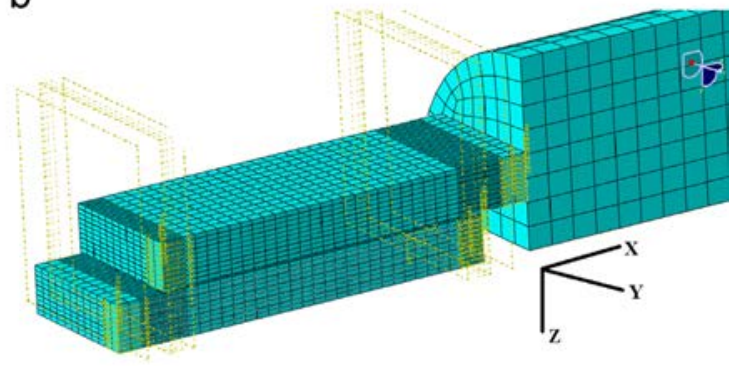

Fig. 3. Numerical mesh of the model: (a) quasi-static case and (b) impact case.

For the quasi-static case, the composite plies were modeled as solid composite layup with 5 integration points each, which means 1 element through thickness contains 10 plies. Each simulation was carried out for $90 \mathrm{~s}$ with results written each $0.5 \mathrm{~s}$.

For the impact case, and since composite layup cannot be used in ABAQUS explicit, each composite ply was modeled as a part having orthotropic material properties with a specified material orientation, and tied to each other which developed the composite laminate. Each simulation was carried out for $40 \mu \mathrm{s}$ with results stored each $0.25 \mu \mathrm{s}$.

\subsection{Average shear stress and homogeneity coefficient}

The objective is to capture the geometrical and material influence on the shear stress distribution in the adhesive layer. For the quasi-static case, stress distributions were extracted at the total time point, which corresponds to the higher displacementstress levels. Meanwhile, stress distributions were extracted at their highest point for the impact-case which does not correspond to the higher displacement value, as will be shown in Section 3.1, Fig. 7. Stress values were extracted at the mid-plane cutting the model into half through the width, and averaged through adhesive thickness. Actually, the average shear stress $\tau_{x y}^{a v}$ should be calculated as:

$\tau_{x z}^{a v}(t)=\frac{1}{L \times T_{a} \times W} \int_{0}^{L} \int_{0}^{W} \int_{0}^{T_{a}} \tau_{x z}(x, y, z, t) d x d y d z$,

where $L, T_{a}$ and $W$ are defined in Fig. 1 as overlap length, adhesive thickness and joint width, respectively. Fortunately, the shear stress is almost constant through the joint width. Consequently, we considered the shear stress at the vertical mid-plane $(y=W / 2)$. Then the average shear stress was approximated by

$\tau_{x z}^{a v}(t) \approx \frac{1}{L \times T_{a}} \int_{0}^{L} \int_{0}^{T_{a}} \tau_{x z}\left(x, y=\frac{W}{2}, z, t\right) d x d z$

The maximum average shear stress used for comparison was defined as the maximum with respect to time of the average shear stress. It reads

$\bar{\tau}_{x z}^{\max }=\max _{t} \tau_{x z}^{a v}(t)$

Additionally to the average shear stress, homogeneity coefficient $\alpha_{\tau_{x z}}(t)$ was defined to quantify the stress heterogeneity in the joint. This coefficient reads

$\alpha_{\tau_{x z}}(t)=\sqrt{\frac{1}{L \times T_{a}} \int_{0}^{L} \int_{0}^{T_{a}} \frac{\left|\tau_{x z}(x, y=(W / 2), z, t)-\tau_{x z}^{a v}(t)\right|^{2}}{\left|\tau_{x z}^{a v}(t)\right|^{2}} d x d z}$

The more this coefficient tends to 0 , the more the shear stress is homogeneous in the adhesive layer.

Specimen geometry, material properties and wave propagation govern the homogeneity of stress distribution [8]. Dynamic effects disappear once the transient regime disappears. In this study, we

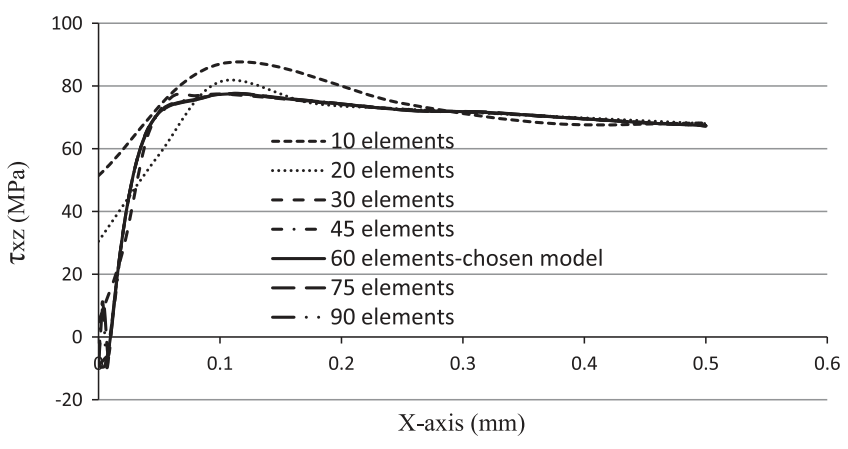

Fig. 4. Convergence curves, edge shear stress in terms of fine-mesh quality.

Table 2

Error\% for different fine-mesh quality configurations.

$\begin{array}{llllllll}\text { Number of elements for the } 0.5 \mathrm{~mm} \text { edge } & 10 & 20 & 30 & 45 & 60 & 75 & 90\end{array}$

$\begin{array}{llllllll}\text { Error (\%) } & 12.4 & 5.0 & 0.5 & 0.1 & 0 & 0 & 0\end{array}$

were interested in the permanent regime which occurs before the maximum shear stress value as will be shown in Fig. 7. Therefore, we focused on the homogeneity coefficient $\alpha_{\tau_{x z}}(t)$ at the instant (time reference) when the shear stress equals its maximum value.

\subsection{Convergence study}

In order to ascertain convergence, a primary mesh convergence study for the entire model was applied. Subsequently, we have focused on the critical region (near edges) because of the high stress gradients in this part of the model. Namely, a very fine mesh was applied at the edges of the adhesive layer since stress peaks and stress gradients occur at these edges. Fig. 4 depicts the shear stress along the adhesive joint for a number of elements ranging from 10 to 90 . We were interested in the peak stress value. It is therefore shown that convergence was obtained for 45 elements. Subsequently, the result obtained by 90 elements were considered as the reference value to calculate the relative errors in terms of the number of elements at joint edge. Table 2 shows that the relative error is less than $0.1 \%$ for a number of elements higher than 45 . Since processing time difference was negligible between 45 and 60 elements, we chose the 60 elements formulation in order to preserve convergence in case if higher peak stress occurs.

\section{Results and discussions}

\subsection{Reference model}

A reference numerical model was first investigated then only one parameter was changed each time for comparison purposes. 
Table 3 shows material and geometrical parameters for reference model.

Figs. 5 and 6 reveal shear stress in the adhesive layer with respect to time and length for impact and quasi-static reference models, respectively. As expected, no wave propagation occurs for quasi-static case, shear stress increases monotonously with displacement and time. On the opposite, multiple oscillations appear for the impact case. Peaks or resonances are due to the multiple wave reflections within the joint. Shear stress reaches maximum around $20 \mu \mathrm{s}$ which corresponds to the duration of the impact pulse. Subsequently, the stress decreases as soon as the energy transmits to the output bar.

This difference in tendency between quasi-static and impact cases is confirmed in Fig. 7. Fig. 7a depicts the average shear stress which increases linearly in the quasi-static case. For the impact case, the average shear stress is monotonously increasing during the impulse load $(<20 \mu \mathrm{s})$. However, the stress increase rate is lower at the first $\mu$ seconds. In this transient period, the left side of the joint is more loaded than the right part, and stress field is highly heterogeneous. This is confirmed by Fig. 7b. Namely, homogeneity coefficient is very high at the beginning of the simulation (first $6 \mu \mathrm{s}$ ); it drops to an asymptotic value. In the quasi-static, the homogeneity coefficient is constant and equal to the asymptotic value of the impact. Actually, heterogeneity is caused either by wave propagation within the specimen (transient heterogeneity) or by the specimen geometry (structural heterogeneity). As observed by Challita and Othman [8], the transient heterogeneity decreases with time and disappears after some $\mu$-seconds (here $\sim 6 \mu \mathrm{s}$ ). The structural heterogeneity is independent of time. For the quasi-static case, heterogeneity is only due to the specimen geometry, meanwhile, for the impact case, transient and structural heterogeneities coexist at the beginning of the simulation.

In this parametric study, we focused on the maximum average shear stress and on the homogeneity coefficient. The average shear stress gives information on the global evolution of the stress state within the joint. On the other hand, the homogeneity coefficient gives valuable information on the evolution of the stress field heterogeneity. By studying the average shear stress, we will have an insight into the sensitivity of the average shear stress state within the joint to the different investigated parameters. The homogeneity coefficient quantifies the distribution of the stress around its average value.

\subsection{Normal stresses inside adherend}

In this section, we were interested in the no failure assumption for the substrates. This study is limited to the reference model. Namely, normal stresses were extracted and compared with limit normal stresses for outer composite substrate. Normal stresses in all the plies are plotted in Fig. 8. Being the fact that the limit stress for composite is $2730 \mathrm{MPa}$, and the peak normal stress is $540 \mathrm{MPa}$, one can assume that no failure occurs inside the composite adherend.

\subsection{Effect of geometrical parameters}

\subsubsection{Adhesive thickness effect}

The influence of the adhesive thickness was investigated by varying the adhesive thickness from $0.05 \mathrm{~mm}$ to $0.25 \mathrm{~mm}$. Fig. 9 shows that both average shear stress and homogeneity coefficient decrease with increasing adhesive thickness. Same tendency observed for both loading cases. It is worth noting that the tendency of the homogeneity coefficient in terms of the adhesive thickness is in line with the observations made by Challita and Othman [8]. The increase in the adhesive thickness reduces its shear stiffness. Hence, it seems that stiffer adhesives enhance the average shear stress and the homogeneity coefficient.

\subsubsection{Adherend thickness effect}

Adherends' thickness effects were investigated and results are presented in Fig. 10. It is shown that the average shear stress increases with the increase of the adherend thickness. On the opposite, stress homogeneity coefficient is decreasing for increasing adherend thickness. This last observation is in line with the results obtained in Ref. [8] with isotropic metallic substrates.

The increase of the adherend thickness induces a decrease in the adherends' shear stiffness. On the opposite, it improves the longitudinal stiffness. Therefore, it seems that the average shear stress increases with increasing adherends' longitudinal stiffness. On the other hand, the homogeneity coefficient increases with increasing adherends' shear stiffness.

\subsubsection{Overlap length}

In a third step, we investigated the influence of the overlap length. The variation of the average shear stress and stress homogeneity in terms of the overlap length are depicted in

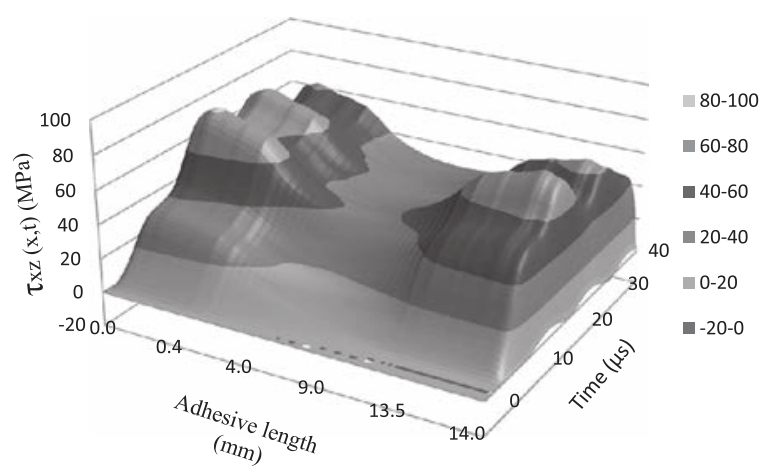

Fig. 5. Shear stress along overlap with respect to time, impact reference model.

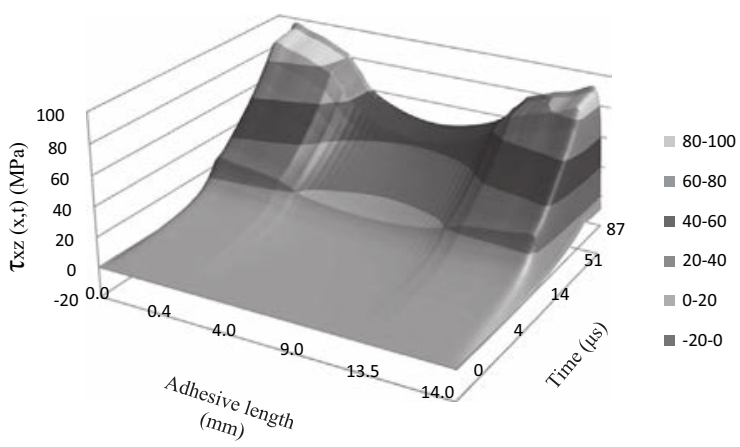

Fig. 6. Shear stress along overlap with respect to time, quasi-static reference model.

Table 3

Reference model, material and geometrical parameters.

\begin{tabular}{|c|c|c|c|c|c|c|c|}
\hline Parameter & $E_{\text {adhesive }}(\mathrm{MPa})$ & Fiber volume (\%) & $T_{\text {adhesive }}(\mathrm{mm})$ & $T_{\text {inner adherend }}(\mathrm{mm})$ & Overlap (mm) & Width (mm) & Orientation \\
\hline Value & 1000 & 60 & 0.1 & 4 & 14 & 12 & 0 (parallel to $X$-axis) \\
\hline
\end{tabular}


a

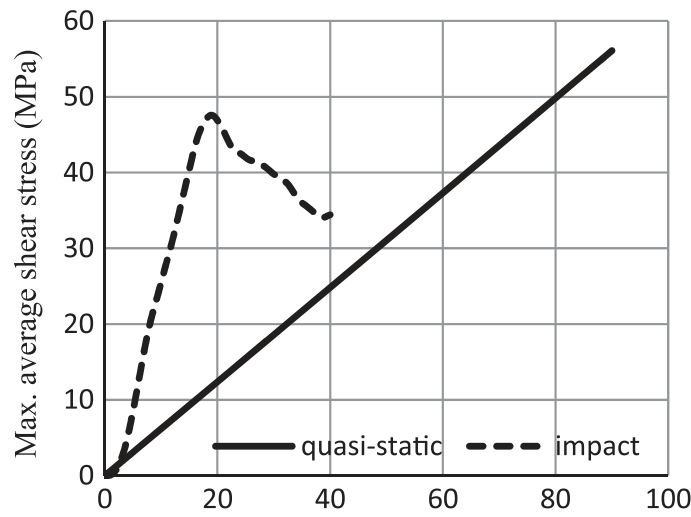

Time, in sec for quasi-static and micro-sec for impact b

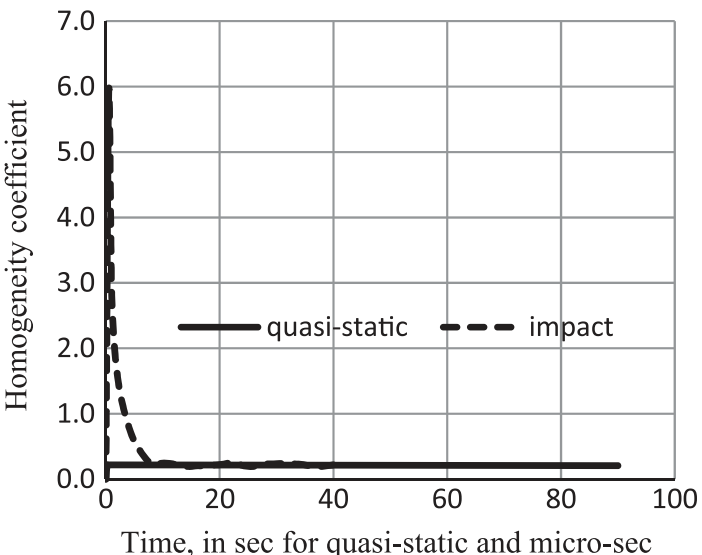

for impact

Fig. 7. Reference model results: (a) average shear stress and (b) homogeneity coefficient in terms of time.

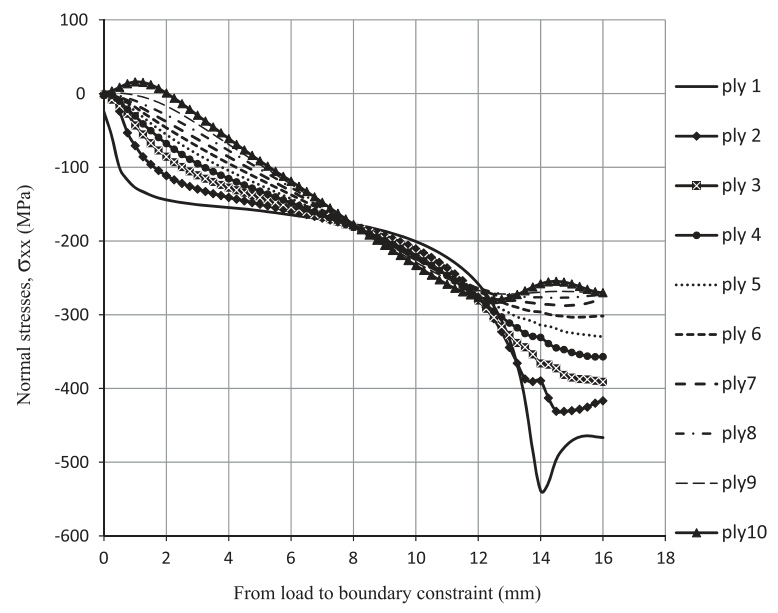

Fig. 8. Normal stresses in the outer adherend in the $X-Z$ plane of symmetry (impact reference model).

Fig. 11. The effects of the overlap length are opposite to the effects of the substrates thickness. This can be explained by the fact that the overlap length has opposite effects on the longitudinal and shear stiffness of the substrates. In other terms, the increase of the overlap length increases the substrates' shear stiffness and decreases the longitudinal stiffness. Hence, the average shear stress and homogeneity coefficient increase respectively with increasing longitudinal and shear stiffness's of the substrates. This observation is in line with the conclusions of Section 3.3.2.

\subsubsection{Width effect}

The last geometrical parameter investigated in this work is joint width. Results are depicted in Fig. 12 which shows that width has almost no effect either on average shear stress nor on stress homogeneity. These results are quite expected as the problem can be assumed to be two-dimensional (Fig. 13).

\subsection{Effect of material parameters}

\subsubsection{Adhesive Young's modulus effect}

Five values of the adhesive Young's modulus were investigated in this section. The parametric study shows that both average shear stress and homogeneity coefficient increase with increasing adhesive Young's modulus. More precisely, stiffer adhesive enhances average shear stress but reduces homogeneity. This result is in line with the conclusion made in Section 3.3.1.

\subsubsection{Adherend fiber volume effect}

In order to investigate the effect of the substrates' stiffness, we considered in this section the influence of the fiber volume percentage. From Fig. 14, it can be concluded that fiber volume has the same effect as the substrate thickness and the opposite effect of the overlap length. The average shear stress increases while homogeneity coefficient decreases with increasing fiber volume. According to mixing law, the longitudinal stiffness increases while the shear stiffness decreases as the fiber volume is augmented. Here also, the average shear stress becomes higher for longitudinally stiffer substrates. On the other hand, the stress heterogeneity in the joint is enhanced as the shear stiffness of the adherends becomes greater.

Challita and Othman [8], showed that the homogeneity coefficient decreases with the isotropic substrate's Young's modulus. It seems here that the shear out-of-plane modulus $G_{13}$ is the most important parameter.

\subsubsection{Effect of substrate orientations}

In order to investigate the effect of anisotropy of substrates, we chose four different configurations for the adherend fiber orientations in addition to the reference one. Fig. 15 illustrates the results. The $0^{\circ}$ orientation is longitudinal parallel to the load, while the laminate $(0,90)$ means the first ply is $0^{\circ}$ then $90^{\circ}$ then $0^{\circ}$ and subsequently, same for the laminate $(45,-45)$.

It was shown that the toughest joint is the joint with fiber parallel to the loading direction and a huge decrease in average shear stress appears when no fiber percentage is parallel to the loading direction. It does not appear that the $45^{\circ}$ fiber is better than the $90^{\circ}$. Indeed, the longitudinal stiffness of substrates is higher for $0^{\circ}$ fibers. Thus, the average shear stress is the best for the highest longitudinal stiffness, i.e., when all fibers are in $0^{\circ}$ direction.

Stress homogeneity coefficient is higher for $45^{\circ}, 90^{\circ}$ or $\left(+45^{\circ}\right)$ $-45^{\circ}$ ). Indeed, $G_{12}$ is higher for these configurations. We have assumed that $G_{12}=G_{13}$. The shear substrates stiffness is higher for $45^{\circ}, 90^{\circ}$ or $\left(+45^{\circ} /-45^{\circ}\right)$. Hence, we can conclude one more time that stress field is more homogeneous for low substrate's shear stiffness. 
a

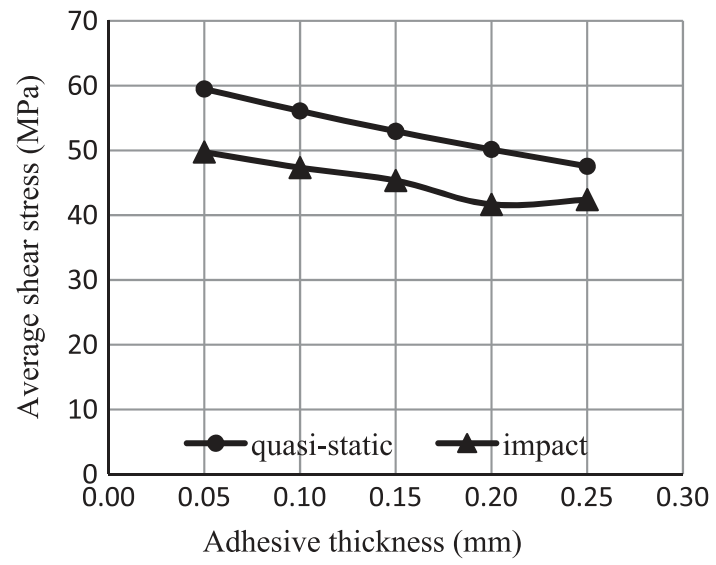

b

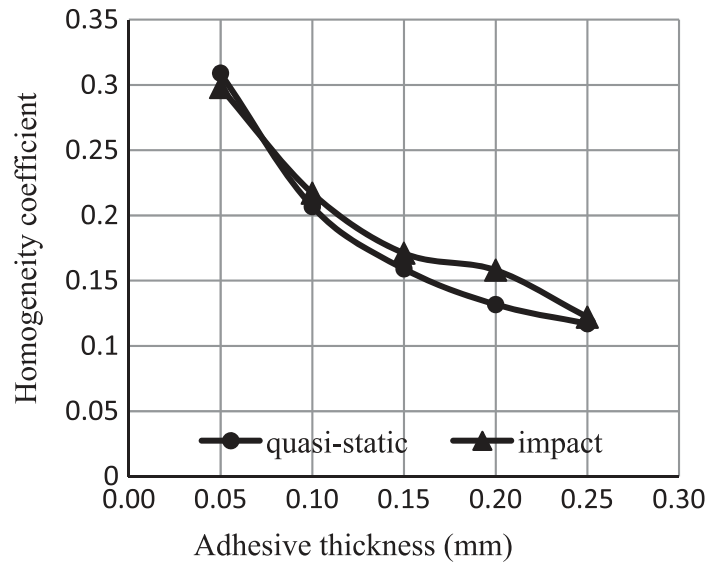

Fig. 9. Effect of the adhesive thickness on average shear stress (a) and homogeneity coefficient (b).

a

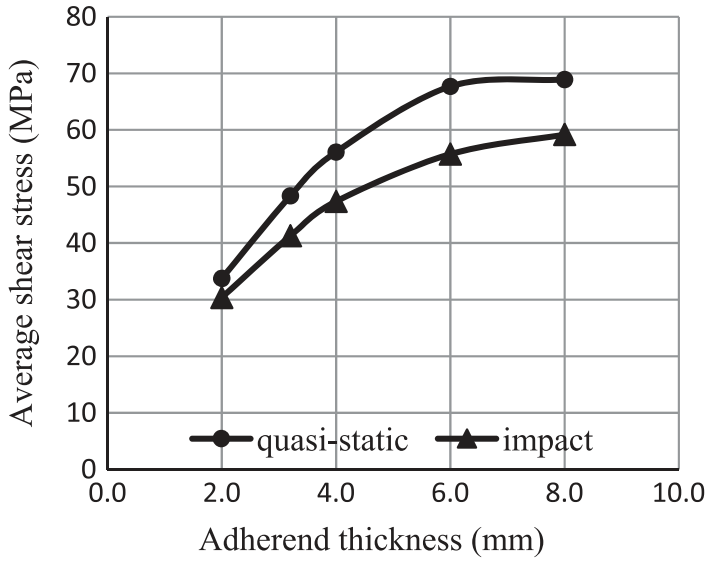

b

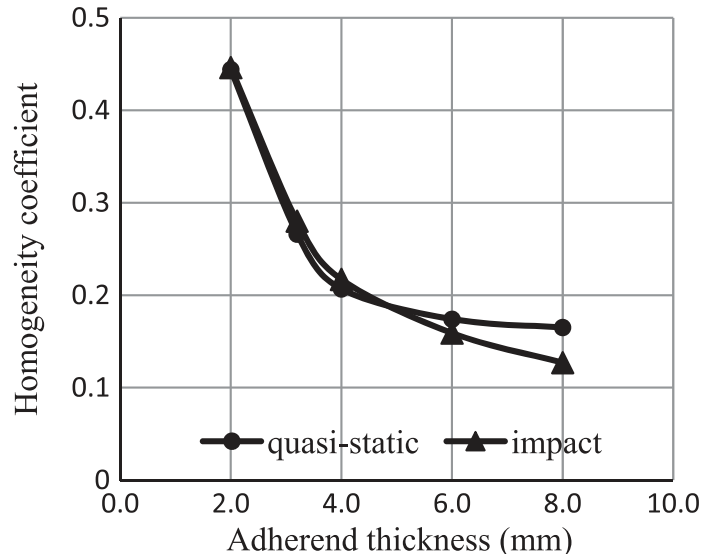

Fig. 10. Effect of the adherend thickness on average shear stress (a) and homogeneity coefficient (b).

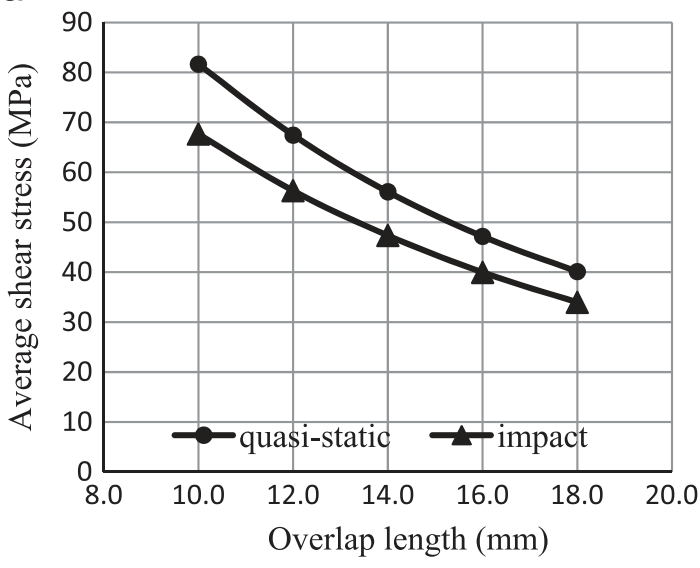

b

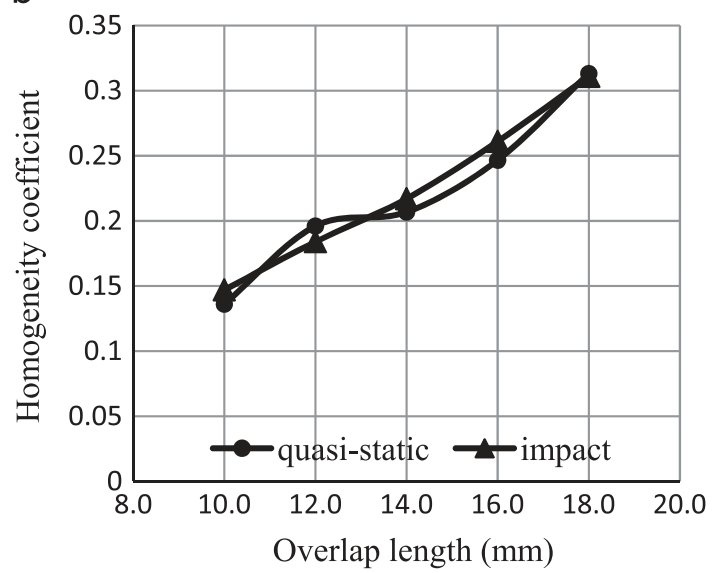

Fig. 11. Effect of the overlap length on average shear stress (a) and homogeneity coefficient (b).

\section{Conclusion}

A parametric three-dimensional finite element study for double lap joints of similar composite adherends under quasi-static and impact loads was accomplished, revealing the geometrical and material influence on the shear stress distribution in the adhesive layer. The stress is due to either structural or wave propagation effects. Structural or geometrical heterogeneity is independent of time. Its contribution does not alter from quasi-static to impact case. On the opposite, heterogeneity due to wave propagation effects was very high at the beginning of the simulation and disappears within some micro-seconds. As we were interested in 
a

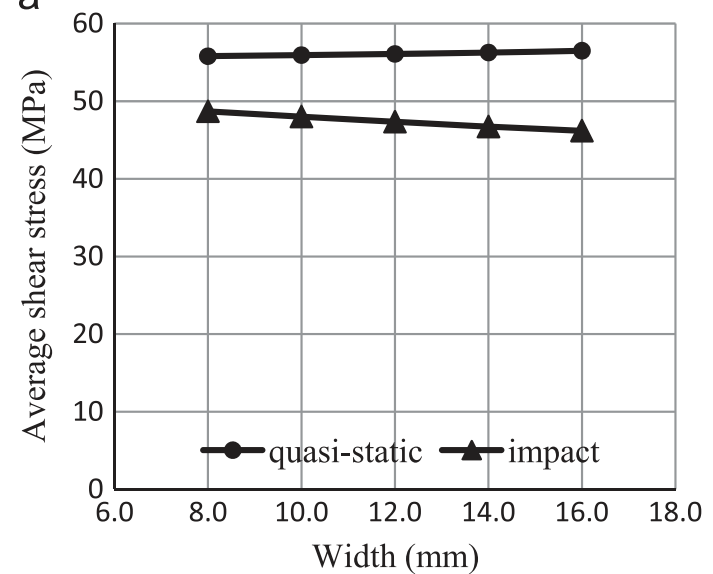

b

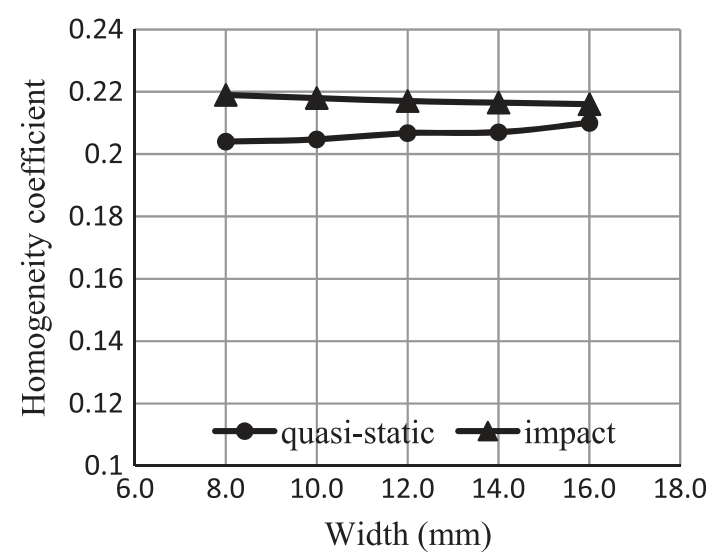

Fig. 12. Effect of the joint width on average shear stress (a) and homogeneity coefficient (b).

a

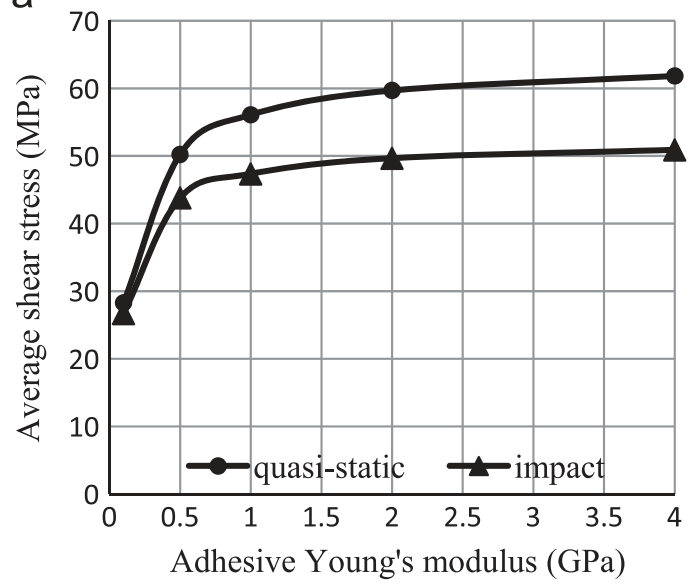

b

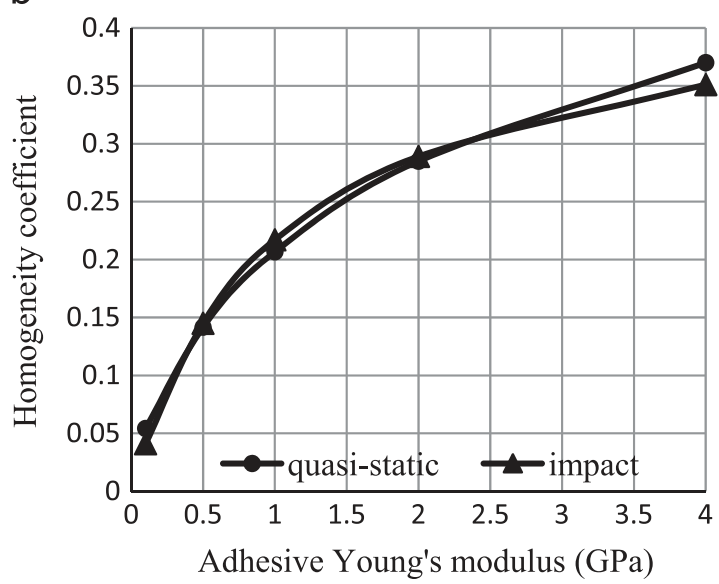

Fig. 13. Effect of the adhesive's Young's modulus on average shear stress (a) and homogeneity coefficient (b).

a

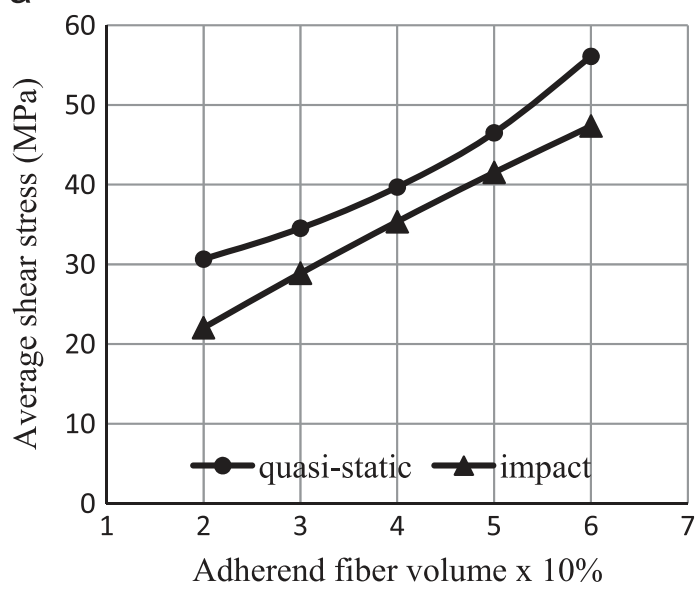

b

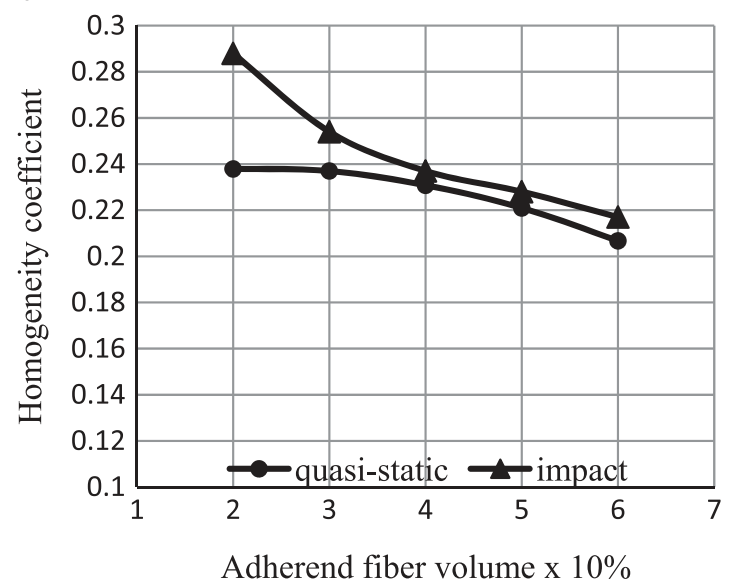

Fig. 14. Effect of the fiber volume on average shear stress (a) and homogeneity coefficient (b).

the permanent regime, only structural effects are influencing stress state in the adhesive joint. Therefore, close results were obtained for both quasi-static and impact cases. For the same imposed displacement, the average shear stress was increasing with increasing substrates' thickness, adhesive Young's modulus and substrates' fiber volume fraction. It was decreasing with increasing adhesive thickness and overlap length. On the other hand, the homogeneity coefficient was increasing with increasing 
a

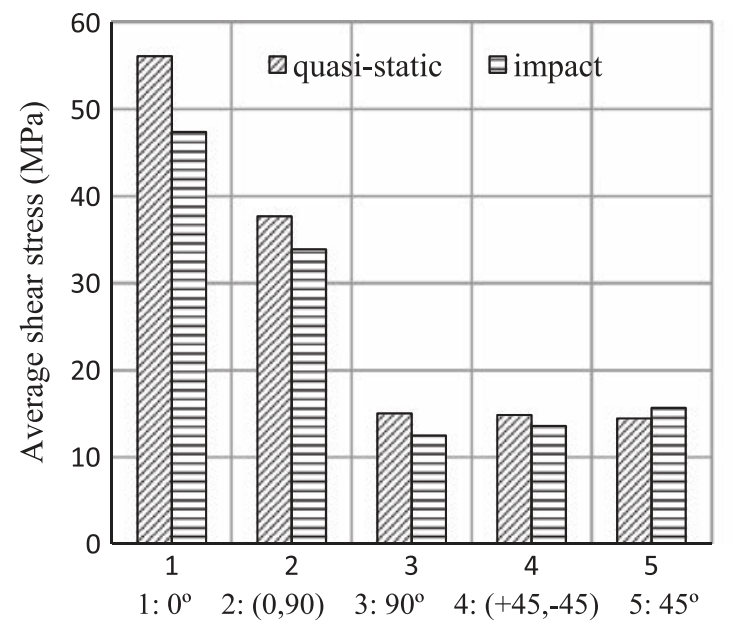

b

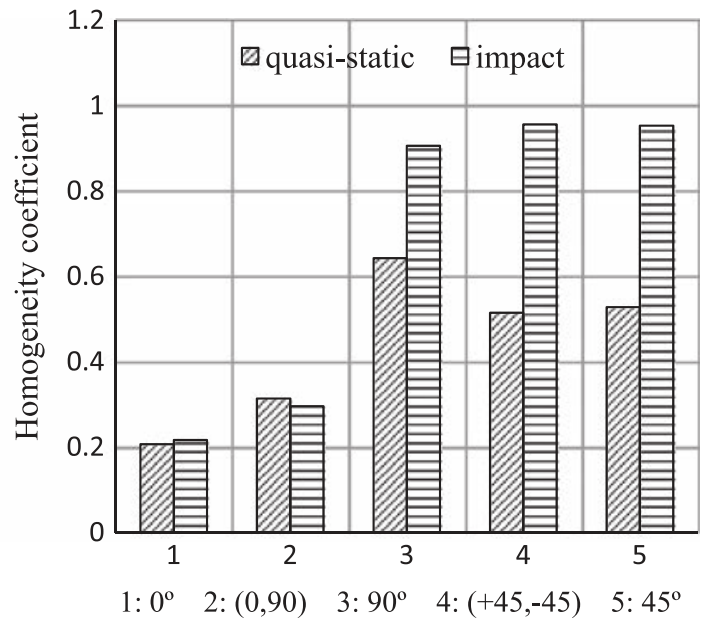

Fig. 15. Effect of fiber orientation on average shear stress (a) and homogeneity coefficient (b).

overlap length and adhesive Young's modulus. It was decreasing with increasing adhesive and substrates' thicknesses and fiber volume fraction. It was also shown that the highest average shear stress is achieved for fibers oriented in the same direction as the load. This orientation yields also to the best stress state homogeneity. These results can be interpreted by the fact that the average shear stress increases with increasing adhesive shear stiffness and substrate longitudinal stiffness; whereas, the stress heterogeneity increases with increasing adhesive and substrate shear stiffness. This is the main finding of this study. Indeed, we succeeded to separate the influence of substrates' longitudinal and shear stiffness as they were considered as orthotropic materials. On the opposite, this can hardly be done by assuming isotropic elastic substrates. Finally, this study was carried out assuming elastic behavior for both adhesive and substrates. Therefore, no failure should occur neither in the adhesive nor in the adherends. Consequently, the results of this work should be understood in this framework.

\section{Acknowledgements}

Khaled Khalil (MMC Team) would like to thank l'Ecole Doctorale des Sciences et Technologies for their collaboration in this work.

\section{References}

[1] Sawa T, Ichikawa K. A stress analysis and strength estimation of stepped lap adhesive joints under static and impact tensile loadings. ASME IMECE 2005:819-25.

[2] Kim H, Kayir T, Mousseau SL. Mechanisms of damage formation in transversely impacted glass-epoxy bonded lap joints. J Compos Mater 2005;39: 2039-52.
[3] Vaidya UK, Gautam ARS, Hosur M, Dutt P. Experimental-numerical studies of transverse impact response of adhesively bonded lap joints in composite structures. Int J Adhes Adhes 2006;26:184-98.

[4] Carlberger T, Stigh U. An explicit FE-model of impact fracture in an adhesive joint. Eng Fract Mech 2007;74:2247-62.

[5] Sawa T, Nagai T, Iwamoto T, Kuramoto H. A study on evaluation of impact strength of adhesive joints subjected to impact shear loadings. ASME IMECE 2008; 15:55-61.

[6] Silberschmidt VV, CasasRodriguez JP, Ashcroft IA. Impact fatigue of adhesive joints. Adv Strength Mater 2009;399:71-8.

[7] Park H, Kim H. Damage resistance of single lap adhesive compoiste joints by transverse ice impact. Int J Impact Eng 2010;37:177-84.

[8] Challita G, Othman R. Finite-element analysis of SHPB tests on double-lap adhesive joints. Int J Adhes Adhes 2010;30:236-44.

[9] Liao L, Sawa T. Finite element stress analysis and strength evaluation of epoxysteel cylinders subjected to impact push-off loads. Int J Adhes Adhes 2011;31: 322-30.

[10] Liao L, Kobayashi T, Sawa T, Goda Y. 3-D FEM stress analysis and strength evaluation of single-lap adhesive joints subjected to impact tensile loads. Int J Adhes Adhes 2011;31:612-9.

[11] Dharan CKH, Hausser FR. Determination of stress-strain characteristics at very high strain rates. Exp Mech 1970;10:370-6.

[12] Villoutreix J, Acetarin JD. Polyétheréthercétone. Techniques de l'ingénieur AM3394.

[13] Guillon D. Fibres de verres de renforcement. Techniques de l'ingénieur A2 110.

[14] Odru P. Calcul et conception des structures composites. Techniques de l'ingénieur A7 792.

[15] Chevalier Y. Comportement élastique et viscoélastique des composites. Techniques de l'ingénieur A7 750.

[16] HUNTSMAN adhesives, Araldite 2031; February 2009.

[17] Higuchi I, Sawa T, Suga H. Three-dimensional finite element analysis of singlelap adhesive joints under impact loads. J Adhes Sci Technol 2002;16: 1585-601.

[18] Higuchi I, Sawa T, Suga H. Three-dimensional finite element analysis of singlelap adhesive joints subjected to impact bending moments. J Adhes Sci Technol 2002; $16: 1327-42$.

[19] Sawa T, Suzuki Y, Kido S. Stress analysis and strength estimation of butt adhesive joints of dissimilar hollow cylinders under impact tensile loadings. J Adhes Sci Technol 2003;17:943-65. 